\title{
Schwerpunkt: Qualitätsmanagement im Bildungswesen
}

\author{
Frieda Heyting/Dieter Lenzen
}

Editorial

Dem klassischen deutschen Bildungsdenken musste die Idee eines Qualitätsmanagements für das Bildungswesen fremd sein. Wenn dort Bildung als Selbstbildung konzipiert war, dann blieb Bildungsqualität unvermeidlich. In ähnlicher Weise konnte sich auch für den Gedanken einer Höherbildung der Menschheit die Frage nach einem diesbezüglichen Management nicht stellen: Der darin aufgehobene Fortschrittsgedanke kennt kein erreichbares Abschlussziel von Qualität, sondern im Fortschrittsgedanken ist Qualitätssteigerung im nicht finalen Sinne immer schon inhärent.

Vielleicht ist diese theoretische Orientierung aus der Frühgeschichte des deutschen Bildungswesens eine der Ursachen dafür, dass die Möglichkeit nie ernsthaft in Betracht gezogen wurde, Bildung könnte, nur recht verstanden, ein Qualitätsdefizit aufweisen. Erst die Resultate internationaler Leistungsvergleiche im Konnex mit dem „empirical turn“ in der deutschen Erziehungswissenschaft drängen den unabweisbaren Verdacht auf, dass die zumindest messbaren Ergebnisse des Lernens in deutschen Schulen verbesserungsfähig sind.

Da das Postulat eines breiten Zugangs der Bevölkerung zu allen Bildungsangeboten es verbietet, das Qualitätsproblem durch Selektion zu lösen, drängt sich die Frage nach der Qualität der Institutionen und der in ihnen stattfindenden Prozesse auf, welche der Aufgabe von Bildung und Ausbildung verpflichtet sind. Diese Tatsache hat es nahe gelegt, in anderen sozialen Teilsystemen nach Verfahren zu suchen, Qualität von Produkten zu sichern. War schon die Übertragung von Verfahren des Qualitätsmanagements aus dem Umkreis von Unternehmen der Güterproduktion auf Dienstleistungsunternehmen nicht ohne Zugeständnisse an die Stringenz der Maßnahmen möglich, so verstärkt sich bei einer Übertragung auf Prozesse des Bildungssystems die Schwierigkeit einer Analogisierung. So ist im Gegensatz zu Produktrohstoffen das „Material“ von Bildungsprozessen nur begrenzt kalkulierbar und im „Bearbeitungsprozess“ noch weniger zu steuern.

Auf der anderen Seite: Wenn Lernen konsequent konstruktivistisch gedacht wird, dann ist die Qualität der Lernumgebung ausschlaggebend für die Effekte des Lernens und die Veranstaltungen des Bildungssystems. Unterrichten und Erziehen ist in diesem Verständnis in erheblichem Maße Gestaltung einer „Lernumwelt“ und kann als solche durchaus als Objekt von Managementprozessen gedacht werden. 
Das vorliegende Heft der Zeitschrift für Erziehungswissenschaft thematisiert das Qualitätsmanagement historisch, vergleichend und bewertend. Im Stichwortartikel „Qualität im Bildungssystem“ werden die begrifflichen Grundlagen des Qualitätsbegriffs erörtert sowie strukturelle Merkmale für das Entstehen der Qualitätsdiskussion im Bildungswesen aufgezeigt (Harm KUPER). Der Beitrag von Hartmut TITZE rekonstruiert den Prozess, der dazu geführt hat, dass das deutsche Bildungsdenken und die dazugehörenden institutionellen Vorkehrungen zur Bedeutungslosigkeit verkommen sind. TITZE zeigt, dass die Herkunft des deutschen Bildungssystems aus dem Absolutismus im Zusammenwirken mit bildungsbürgerlichen Vorstellungen ein rechtzeitiges Umschalten auf Bildungsbeteiligung als nationale Ressource verunmöglicht hat.

Aus der Sicht der Unternehmensberatung fassen Gustav GREvE und Iris PFEIFFER die Grundlinien des Qualitätsmanagements in Unternehmen zusammen und verdeutlichen, dass Verfahren wie Total Quality Management, Benchmarking und Balanced Scorecard in jeder Art von Unternehmen an Bedeutung eher gewinnen als verlieren werden. Die Analogie zu einem Verständnis von Bildungseinrichtungen als Unternehmen wird dadurch gestärkt.

In welchem Maße diese Herausforderungen außerhalb Deutschlands aufgenommen worden sind, zeigen die folgenden Beiträge. Japan, ein Land, welches seit der Meiji-Zeit das preußische Bildungswesen kopierte, hat die Herausforderung angenommen und arbeitet an der Implementation entsprechender Verfahren (Isao KUROSAKI). Für ein historisch auf andere Weise vergleichbares Land, für die Republik Österreich, zeigt Peter PosCH den Stand der Erfahrungen mit dem Qualitätsmanagement im Bildungswesen.

Kritisch nähert sich Maarten SIMONS dem Qualitätsmanagement im Bildungswesen als einer Obsession. Er problematisiert die Konzeptionierung des Arbeitnehmers als Unternehmer seiner selbst in einem auf Qualitätsprozesse zugespitzten Bildungswesen. Der Beitrag fokussiert das Risiko, Lernen als einen Bestandteil von Unternehmerschaft über den Umweg von Qualitätsmanagement zu definieren.

Prozesse der Evaluierung, des Benchmarkings und des Qualitätsmanagements haben in den zurückliegenden Jahren innerhalb des deutschen Bildungssystems zuerst den Hochschulbereich ergriffen. Stefan HORNBOSTEL und Edwin KEINER analysieren, bezogen auf das Fach Erziehungswissenschaft, die Resultate von Hochschulrankings auch im interdisziplinären Vergleich. Der Beitrag öffnet Perspektiven für eine Weiterentwicklung standardisierter Indikatoren im Rahmen der Erziehungswissenschaft.

In der Sammelrezension zum Thementeil des vorliegenden Heftes bespricht HeinzGünter HOLTAPPELS einschlägige Literatur zum Thema „Qualitätsmanagement im Bildungswesen“. 